\title{
Using Team-based Learning to Develop Consultation Skills
}

Authors: Simon Tweddell and John Purvis. Bradford School of Pharmacy, University of Bradford

Background: TBL was introduced into a year 4 Consultation Skills module designed to develop knowledge and skills to consult with patients presenting with minor ailments in community pharmacy. We knew TBL could be used to develop and apply knowledge, could it be used in an innovative way to develop consultation skills? To introduce and evaluate an engaging and interactive set of workshops, developed using TBL principles that aim to develop students': consultation skills; ability to provide peer feedback; involvement in the setting of assessment criteria; skills in evaluating consultation skills.

Description: Students were allocated to teams of 5-6 students based upon experiences of community pharmacy. They worked together during the semester developing knowledge and understanding of the diagnosis and management of minor ailments. Towards the end of the semester, each student was recorded individually consulting with a standardised patient presenting in a pharmacy with a minor ailment. In teams, students reviewed recordings of team members and selected the best to put forward to the 'final' a week later. For the final, the year was divided in half. Teams initially developed the assessment criteria against which they would judge each consultation. This was followed by whole-class discussion, facilitated by tutors and the final criteria agreed. Students watched the recordings of the 8 team's nominated consultation from the other half of the year group, assessed them against the agreed criteria and selected the 'best of the best.' Teams were asked to justify their choices. This was repeated with the other half of the year. The workshops were evaluated qualitatively via student and staff feedback through specific questions on the module evaluation questionnaire and by discussions with staff. Quantitative OSCE performance data was collected and compared with previous cohorts

Results: Students really enjoyed the recording and initial review and selection within their team. The class perceptions of the 'final' were also very positive. The course review forms and feedback received during the sessions demonstrated that both sessions were well received by students. We had to curb discussion and debate due to time constraints. More time will be dedicated to the final class in subsequent years. Comparison of assessment data showed a small increase in OSCE performance compared to previous cohorts.

Conclusion: Team-based learning principles can be used for developing Consultation Skills in pharmacy students. 